\title{
Docetaxel-associated myalgia-arthralgia syndrome in patients with breast cancer
}

This article was published in the following Dove Press journal:

Breast Cancer - Targets and Therapy

23 January 2017

Number of times this article has been viewed

\author{
Chelsea Seguin' \\ Natalie Kovacevich' \\ loannis A Voutsadakis ${ }^{2,3}$ \\ 'Clinical Trials Unit, ${ }^{2}$ Division of \\ Medical Oncology, Department of \\ Internal Medicine, Sault Area Hospital, \\ Sault Ste. Marie, ${ }^{3}$ Division of Clinical \\ Sciences, Northern Ontario School of \\ Medicine, Sudbury, ON, Canada
}

Correspondence: loannis A Voutsadakis Division of Medical Oncology, Department of Internal Medicine, 750 Great Northern Road, Sault Area Hospital, Sault Ste. Marie, ON P6B 0A8, Canada

Tel +l 7057593434

Email ivoutsadakis@yahoo.com; ivoutsadakis@nosm.ca
Background: As taxanes are increasingly used in oncology, the myalgia-arthralgia syndrome (M-AS) that represents an adverse effect of these drugs is becoming more common. Nevertheless, information regarding predisposing factors, prevention, and therapy of the syndrome is still lacking.

Patients and methods: Women who had received docetaxel as part of the FEC-D(T) regimen for the adjuvant treatment of breast cancer were retrospectively identified from the records of our oncology department. Data on demographics, disease specifics, adverse effects, and treatment were reviewed. Patients were divided into two groups: those who developed M-AS after docetaxel treatment and those who did not develop the syndrome. The two groups were compared to identify risk factors for M-AS. Effectiveness of drugs used for M-AS was evaluated. Results: Sixty-seven patients were identified as fulfilling the inclusion criteria. Nineteen patients developed the M-AS after the first docetaxel administration. Forty-eight patients did not develop the syndrome. Three patients in this group were excluded because they had been taking gabapentin or pregabalin at the time of docetaxel administration for another indication. The remaining 45 patients constituted the control group. The two groups were similar in age, menopause status, stage of their cancer, and histology. The M-AS group had a higher median body surface area and was more likely to receive less than the three intended cycles of docetaxel. Nonsteroidal anti-inflammatory drugs, atypical antiepileptics, extended corticosteroids, and opioids were drugs used as M-AS treatments.

Conclusion: Docetaxel-associated M-AS is an adverse effect causing incomplete drug treatment. Possible risk factors and effectiveness of treatments for the syndrome are presented.

Keywords: myalgia-arthralgia syndrome, taxanes, gabapentin, pregabalin, adverse effects

\section{Introduction}

Taxane-based chemotherapy regimens constitute one of the most widely used cytotoxic antineoplastic treatments for various cancers including breast, gynecologic, lung, and genitourinary malignancies. Taxanes have a well-known toxicity profile that includes bone marrow suppression, hypersensitivity reactions, skin toxicities, dose-limiting peripheral sensory neuropathy, and alopecia. ${ }^{1}$ The taxane-induced myalgia-arthralgia syndrome (M-AS) is a less well-understood toxicity, and it is distinct from taxaneassociated peripheral neuropathy. M-AS presents most often with moderate or severe generalized musculoskeletal pain the first hours or days after taxane administration. Although in our clinical experience, severe pain after taxane administration is commonly encountered as a factor leading to patients' requests for discontinuation of 
treatment, no data exist on the incidence of this occurrence that could decrease the overall efficacy of oncologic treatment. There is no consensus with regard to the treatment of this adverse effect. Various pharmacologic agents have been anecdotally reported as useful in treating the syndrome, but no randomized data exist. ${ }^{2,3}$

We performed a retrospective review of the records in our clinic to identify patients treated with docetaxel for localized breast cancer who experienced taxane-induced M-AS so as to investigate possible risk factors for developing the syndrome and the effect that the syndrome has in delivering the scheduled dose of docetaxel, and to evaluate drug treatments.

\section{Patients and methods}

Case records of women with localized breast cancer treated in our center over a 4-year period were retrospectively reviewed. Further information was collected from records of patients with localized breast cancer who received docetaxel treatment in the adjuvant or neoadjuvant setting. Data on patients' age, menopause status, body surface area (BSA), clinical presentation, biologic characteristics of the tumors, comorbidities, and concomitant medications were recorded. BSA is calculated from the height and weight of patients and forms the basis for calculation of the dose of the chemotherapy administered in each patient. Data on the chemotherapy treatment regimen (ie, 5-fluorouracil, epirubicin, cyclophosphamide-docetaxel [FEC-D] with or without trastuzumab), number of cycles, docetaxel dose, use of granulocyte colony-stimulating factor (G-CSF), and patient recorded pain (Edmonton Symptom Assessment Scale [ESAS] or other reported pain in a scale or descriptive manner in a medical or nursing note) associated with chemotherapy cycle were also extracted.

A total of 102 breast cancer patients who received docetaxel in the (neo)adjuvant setting from 2011 to 2015 were identified. After initial review, 26 patients were excluded because of incomplete documentation of adverse effects in their records, leaving 76 patients for further chart evaluation. Nine patients were further excluded because of the presence of a chronic pain syndrome antecedent to chemotherapy and with no increase after docetaxel use or because of first use of G-CSF concomitant with the first docetaxel cycles, making assertion or exclusion of docetaxel-associated M-AS impossible. Thus, 67 patients were included in this review (Figure 1).

Pain evaluations were performed using the ESAS. ESAS is a valid and reliable assessment tool for the assessment of nine common symptoms experienced by cancer patients. ${ }^{4}$ Pain, graded on a scale of 0 (no pain) to 10 (worst possible

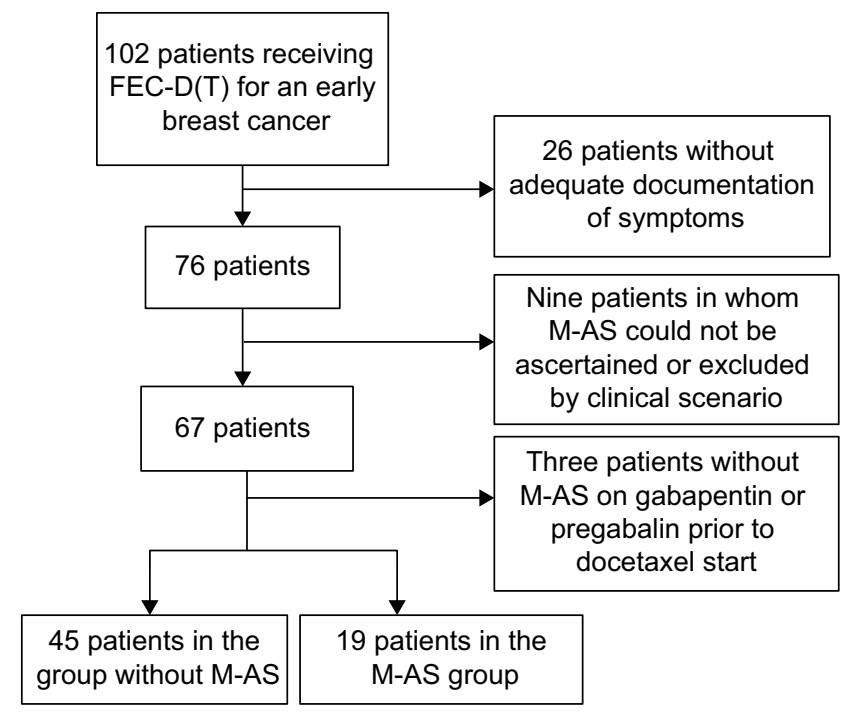

Figure I Patient flowchart.

Note: FEC-D(T): 5-fluorouracil, epirubicin, cyclophosphamide followed by docetaxel with or without trastuzumab.

Abbreviation: M-AS, myalgia-arthralgia syndrome.

pain), is one of the symptoms assessed on the ESAS tool and was used in combination with descriptive clinical assessments for determining the development of taxane-induced M-AS. Patients' ESAS scores for pain were recorded at each cycle of their FEC and docetaxel treatment. Patients were considered to have the M-AS if they developed musculoskeletal pain of more than 3 in the scale in the absence of previous pain or if their pain increased for at least two numbers in the scale if pain was present before the docetaxel administration (eg, due to previous G-CSF use).

The effectiveness of treatment of M-AS was based on a combination of decreased ESAS pain scores from Cycle 4 to Cycle 5, as well as additional clinical documentation provided in the chart notes of treatment efficacy or reduced pain in subsequent cycles. The effectiveness of treatment was defined as complete if there was a disappearance of pain or decrease to mild (ESAS Scores 1-3) if initially severe (ESAS Scores 7-10). Partial effectiveness was defined as decrease of the pain intensity from severe to moderate (ESAS Scores 4-6) or from moderate to mild.

The $\chi^{2}$ test was used for the statistical comparison of categorical variables in the two groups of patients, ie, without M-AS (control) and with M-AS. The two-tailed $t$-test was used for comparison of means of continuous variables. Statistics were performed using calculators available online (http://www.socscistatistics.com and http://graphpad.com).

The study has been approved by the Ethics Committee of Sault Area Hospital, Ontario, Canada. In view of the retrospective nature of the study, no individual patient consent was required. 


\section{Results}

\section{Characteristics and predictive factors of M-AS}

All 67 patients reviewed with localized breast cancer were treated with FEC-D(T) chemotherapy regimen (three cycles of 5-fluorouracil (5FU), epirubicin, and cyclophosphamide followed by three cycles of docetaxel at a dose of $100 \mathrm{mg} / \mathrm{m}^{2}$ with or without trastuzumab). Nineteen patients developed M-AS following their first dose of docetaxel (Cycle 4 of FEC$\mathrm{D}(\mathrm{T}))$. Of the 48 patients who did not develop the syndrome, three patients had been taking gabapentin or pregabalin at the time of their docetaxel treatment and hence are described separately. The remaining 45 patients were included in the control group for the current analysis. Tables 1 and 2 outline the characteristics of the M-AS and control group of patients and include details such as age, BSA, characteristics of the tumor (stage, histology, hormone receptor status), setting of treatment (neoadjuvant or adjuvant), and laboratory values (total bilirubin and calculated glomerular filtration rate as possible surrogates of drug metabolism capacity and platelet counts as a surrogate for baseline inflammatory status). Among all these, BSA was found to be higher in the M-AS group (mean 1.85 vs 1.71 in the control group using $\chi^{2}$ test, $p=0.013$; Table 1$)$.

Other baseline characteristics of the patients in the two groups as well as concomitant medications or medical conditions that may play a role or may be associated with the development of M-AS were evaluated (Table 2). Development of peripheral neuropathy after docetaxel administration was more prevalent in the M-AS group $(p=0.021)$, whereas the presence of a rheumatologic diagnosis (mainly osteoarthritis) or diabetes mellitus was not. Use of G-CSF at the start of docetaxel treatment (Cycle 4) was not associated with the M-AS ( $p=0.12)$. G-CSF had to have been started in prior cycles because start at Cycle 4 was an exclusion criterion. Use of statins, known to be associated with myalgias, or use of antidepressants was also not different in the two groups.

Sixteen of the 64 patients $(25 \%)$ required a dose reduction or discontinuation of docetaxel (Table 3). In the group that did not experience M-AS, there were nine cases $(20 \%)$ that required dose reduction or discontinuation of docetaxel, with the most prominent reason being skin toxicity (including palmar-plantar syndrome), followed by fatigue, gastrointestinal toxicity, and neutropenia. In the group that experienced M-AS, there were seven cases (37\%) that required a dose reduction or opted for discontinuation of docetaxel, of which in five patients $(26 \%)$ these were due to the arthralgia-
Table I Baseline characteristics of patients in the series

\begin{tabular}{|c|c|c|c|c|}
\hline & $\begin{array}{l}\text { All } \\
\text { patients }\end{array}$ & $\begin{array}{l}\text { Control } \\
\text { group }\end{array}$ & $\begin{array}{l}\text { M-AS } \\
\text { group }\end{array}$ & $t$-test $/ \chi^{2}$ \\
\hline$N(\%)$ & $64(100)$ & $45(70.3)$ & $19(29.7)$ & \\
\hline BSA mean (SD) & & I.7I (0.2I) & $1.85(0.15)$ & $p=0.013$ \\
\hline \multicolumn{5}{|l|}{ Age at diagnosis } \\
\hline Mean (SD) & $56(24-80)$ & $57.6(10.7)$ & $56.3(9.2)$ & $p=0.65$ \\
\hline$>65$ years & $15(23.4)$ & $12(26.7)$ & $3(15.8)$ & $p=0.34$ \\
\hline$\leq 65$ years & $49(76.6)$ & $33(73.3)$ & $16(84.2)$ & \\
\hline \multicolumn{5}{|l|}{ Menopause status } \\
\hline Pre-I & $25(39.1)$ & $19(42.2)$ & $6(31.6)$ & $p=0.42$ \\
\hline perimenopausal & & & & \\
\hline Postmenopausal & 39 (60.9) & $26(57.8)$ & $13(68.4)$ & \\
\hline \multicolumn{5}{|l|}{$E R / P R$} \\
\hline $\begin{array}{l}\text { Positive (either } \\
\text { or both) }\end{array}$ & $48(75)$ & $31(68.9)$ & $17(89.5)$ & $p=0.08$ \\
\hline Negative (both) & $16(25)$ & 14 (3I.I) & $2(10.5)$ & \\
\hline \multicolumn{5}{|l|}{ HER-2 } \\
\hline Positive & $17(26.6)$ & $12(26.7)$ & $5(26.3)$ & $p=0.97$ \\
\hline Negative & $47(73.4)$ & $33(73.3)$ & 14 (73.7) & \\
\hline \multicolumn{5}{|l|}{ Stage } \\
\hline I & $8(12.5)$ & $7(15.5)$ & I (5.3) & III vs I and II \\
\hline II & $45(70.3)$ & 31 (68.9) & 14 (73.7) & $p=0.49$ \\
\hline III & II (I7.2) & $7(15.5)$ & $4(21.0)$ & \\
\hline \multicolumn{5}{|l|}{ Histology } \\
\hline Ductal & $56(87.5)$ & 39 (86.7) & $17(89.5)$ & $p=0.75$ \\
\hline Lobular/mixed & $8(12.5)$ & $6(13.3)$ & $2(10.5)$ & \\
\hline \multicolumn{5}{|l|}{ Treatment } \\
\hline Neoadjuvant & I8 (28.I) & $12(26.7)$ & $6(3 \mid .6)$ & $p=0.68$ \\
\hline Adjuvant & $46(7 \mid .9)$ & $33(73.3)$ & $13(68.4)$ & \\
\hline
\end{tabular}

Notes: Column "All patients" includes the whole series of patients including those who developed M-AS and those who did not. $\chi^{2}$ test between the group without M-AS (control) and the group with M-AS. Wherever there are two or more categories, the grouping of the comparison is mentioned in the same column. The $\chi^{2}$ and the two-tailed $t$-test were performed for comparisons of rates and means, respectively.

Abbreviations: M-AS, myalgia-arthralgia syndrome; BSA, body surface area; ER/ PR, estrogen receptor/progesterone receptor; HER-2, human epidermal growth factor receptor 2 .

myalgia pain (one patient of these five had also developed febrile neutropenia) (Table 3). Patients with M-AS were significantly more likely to discontinue docetaxel treatment (four patients [21.1\%] compared with two patients [4.4\%] in the control group, $\chi^{2}=4.33, p=0.033$ ).

Cycle 4 of the FEC-D protocol involves the introduction of docetaxel chemotherapy, and the M-AS group had a significantly higher mean pain at this point in their treatment (Figure 2). The mean ESAS pain score in the M-AS group was 7 with standard deviation (SD) of 2.23 vs a mean of 1.13 with SD of 1.76 in the control group (two-tailed $t$-test $=11.2$, $p<0.0001)$.

\section{Treatment of M-AS}

Of the 19 patients who developed M-AS following docetaxel infusion, three patients were prescribed the atypical antiepileptics gabapentin (one patient) and pregabalin (two 
patients). The two patients treated with pregabalin (both at $25 \mathrm{mg}$ three times a day) had a complete and partial response of their pain. Extended use of corticosteroids, beyond Day 2 that is the standard premedication regimen of docetaxel, was another commonly encountered M-AS therapy and was prescribed in six patients (in one patient together with opioids). It had a complete or partial effectiveness in five of the six patients. Six more patients were treated with opioids, nonsteroidal anti-inflammatory drugs (NSAIDs), acetaminophen, or combinations thereof. The four patients who had opioids included in their treatment seemed to have a better response than the two patients treated with acetaminophen or NSAIDs (ibuprofen) alone. Four patients did not receive any specific analgesic treatment for their M-AS (three of them were given prescriptions that did not fill and the fourth did not report the syndrome and decided to discontinue further treatment).

Three patients were excluded from the group comparisons because they were chronically taking gabapentin

Table 2 Possible associations of M-AS with patients' concomitant conditions, medications use, and laboratory values

\begin{tabular}{|c|c|c|c|c|}
\hline & $\begin{array}{l}\text { All patients } \\
\text { (\%) }\end{array}$ & $\begin{array}{l}\text { Control } \\
\text { group (\%) }\end{array}$ & $\begin{array}{l}\text { M-AS group } \\
\text { (\%) }\end{array}$ & $\chi^{2} / t$-test \\
\hline $\mathrm{N}(\%)$ & $64(100)$ & $45(70.3)$ & $19(29.7)$ & \\
\hline \multicolumn{5}{|c|}{ Peripheral neuropathy } \\
\hline Yes & $15(23.4)$ & $7(15.6)$ & $8(42.1)$ & $p=0.021$ \\
\hline No & $49(76.6)$ & $38(84.4)$ & II (57.9) & \\
\hline \multicolumn{5}{|c|}{ Rheumatologic diagnosis } \\
\hline Yes & $23(35.9)$ & $14(31.1)$ & $9(47.4)$ & $p=0.21$ \\
\hline No & $4 I(64 . I)$ & 31 (68.9) & $10(52.6)$ & \\
\hline \multicolumn{5}{|l|}{ Diabetes mellitus } \\
\hline Yes & $7(10.9)$ & $4(8.9)$ & $3(15.8)$ & $p=0.4 \mathrm{I}$ \\
\hline No & $57(89.1)$ & 4I (9I.I) & $16(84.2)$ & \\
\hline \multicolumn{5}{|c|}{ G-CSF use at Cycle 4} \\
\hline Yes & $31(48.4)$ & $19(42.2)$ & $12(63.2)$ & $p=0.12$ \\
\hline No & $33(51.6)$ & $26(57.8)$ & $7(36.8)$ & \\
\hline \multicolumn{5}{|l|}{ Statin use } \\
\hline Yes & $13(20.3)$ & $9(20.0)$ & $4(2 I .1)$ & $p=0.92$ \\
\hline No & $51(79.7)$ & $36(80.0)$ & $15(78.9)$ & \\
\hline \multicolumn{5}{|l|}{ Antidepressant use } \\
\hline Yes & $10(15.6)$ & $7(15.6)$ & $3(15.8)$ & $p=0.98$ \\
\hline No & $54(84.4)$ & $38(84.4)$ & $16(84.2)$ & \\
\hline $\begin{array}{l}\text { Total bilirubin } \\
\text { mean (SD) }\end{array}$ & & $9.23(3.43)$ & $7.47(2.89)$ & $p=0.055$ \\
\hline $\begin{array}{l}\text { Serum creatinine } \\
\text { mean (SD) }\end{array}$ & & $65.2(10.6)$ & $70.1(10.8)$ & $p=0.09$ \\
\hline cGFR mean (SD) & & $82.0(14.0)$ & $77.2(12.7)$ & $p=0.2$ \\
\hline $\begin{array}{l}\text { Platelet count } \\
\text { mean (SD) }\end{array}$ & & $245(60)$ & $249(49)$ & $p=0.99$ \\
\hline
\end{tabular}

Notes: The $\chi^{2}$ test was used to compare rates, and the two-tailed $t$-test was used to compare means.

Abbreviations: M-AS, myalgia-arthralgia syndrome; cGFR, calculated glomerular filtration rate; G-CSF, granulocyte colony-stimulating factor; SD, standard deviation.
Table 3 ESAS pain score and number of patients and reason for dose adjustment or discontinuation of docetaxel

\begin{tabular}{|c|c|c|c|c|}
\hline & $\begin{array}{l}\text { All patients } \\
\text { (\%) }\end{array}$ & $\begin{array}{l}\text { Control } \\
\text { group (\%) }\end{array}$ & $\begin{array}{l}\text { M-AS } \\
\text { group (\%) }\end{array}$ & $\chi^{2} / t$-test \\
\hline \multicolumn{5}{|l|}{$\begin{array}{l}\text { at Cycle } 4 \text { (mean } \\
{[S D] \text { ) }}\end{array}$} \\
\hline \multicolumn{5}{|c|}{ Docetaxel dose adjustment } \\
\hline No & $48(75.0)$ & $36(80.0)$ & $12(63.2)$ & $p=0.15$ \\
\hline Yes & $16(25.0)$ & $9(20.0)$ & $7(36.8)$ & \\
\hline \multicolumn{5}{|c|}{ Number of docetaxel cycles completed } \\
\hline I or 2 & $6(9.4)$ & $2(4.4)$ & $4(21.1)$ & $p=0.033$ \\
\hline 3 & $58(90.6)$ & $43(95.6)$ & 15 (78.9) & \\
\hline \multicolumn{5}{|c|}{ Reasons for dose decrease or discontinuation of docetaxel } \\
\hline $\begin{array}{l}\text { Myalgia- } \\
\text { arthralgia }\end{array}$ & $5(7.8)$ & $0(0)$ & $5(7 \mid .4)$ & $\begin{array}{l}\text { Myalgia } \\
\text { vs other }\end{array}$ \\
\hline $\begin{array}{l}\text { Severe infusion } \\
\text { reaction }\end{array}$ & $\mathrm{I}(\mathrm{I} .6)$ & $\mathrm{I}(\mathrm{II} . \mathrm{I})$ & $0(0)$ & $p=0.002$ \\
\hline Neutropenia & $\mathrm{I}(\mathrm{I} .6)$ & $0(0)$ & $2(28.6)$ & \\
\hline Fatigue & $4(6.2)$ & $3(33.3)$ & I (14.3) & \\
\hline Renal toxicity & I (I.6) & I (II.I) & $0(0)$ & \\
\hline Skin toxicity & $3(4.7)$ & $3(33.3)$ & $0(0)$ & \\
\hline GI toxicity & $2(3.1)$ & $2(22.2)$ & $0(0)$ & \\
\hline $\begin{array}{l}\text { Total with } \\
\text { toxicity (\%) }\end{array}$ & $16(25.0)$ & $9(20.0)$ & $7(36.8)$ & \\
\hline
\end{tabular}

Notes: Total number of patients with toxicities exceeds 100\% because one patient in the control group had both fatigue and nausea with vomiting and one patient with M-AS had febrile neutropenia in addition to myalgia-arthralgia as the reason of adjustment/discontinuation. The $\chi^{2}$ test was used to compare rates, and the twotailed $t$-test was used to compare means.

Abbreviations: ESAS, Edmonton Symptom Assessment Scale; M-AS, myalgiaarthralgia syndrome; Gl, gastrointestinal; SD, standard deviation.

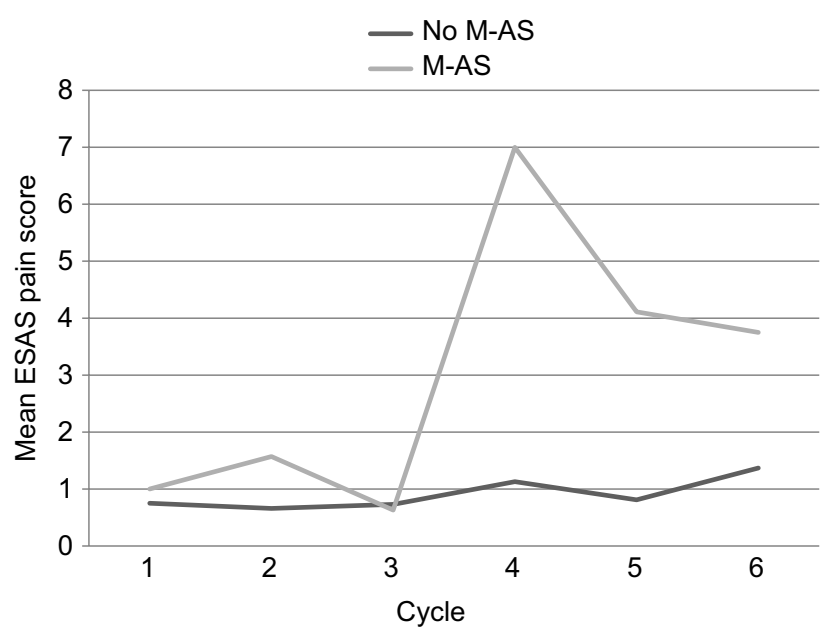

Figure 2 Average ESAS pain score for patients with and without M-AS. Note: Cycle 4 is the first cycle of docetaxel.

Abbreviations: ESAS, Edmonton Symptom Assessment Scale; M-AS, myalgiaarthralgia syndrome.

(two patients) or pregabalin (one patient) at the time of their docetaxel treatment for other indications. No M-AS or increase of preexisting pain was documented in any of these three patients. 


\section{Discussion}

M-AS is a condition consisting of generalized myalgias and arthralgias that follow the administration of taxanes. It usually appears within the 48 hours after taxane infusion, and, although varying in severity, it can be quite debilitating in a significant proportion of patients, often being the most prominent adverse effect. With the incorporation of taxanes in various chemotherapeutic regimens, including adjuvant regimens for breast cancer, the syndrome currently constitutes an increasingly frequent medical problem. Despite its common occurrence and possible clinical importance, M-AS has not been addressed in the medical literature beyond a few case reports and small series. ${ }^{2,3}$ In this regard, our current report represents the first attempt of studying M-AS in a more extensive series of patients and a more systematic way.

The pathophysiology of the syndrome has not been completely elucidated, but it is believed that it is related to the peripheral neuropathy that is a more chronic adverse effect of taxane chemotherapeutics, resulting from peripheral nerve toxicity or related to the central perception of pain. ${ }^{5}$ In this regard, the occurrence and severity of the syndrome has been reported to correlate with the subsequent development of peripheral neuropathy. ${ }^{6}$ Our data agree with this observation as peripheral neuropathy was more commonly seen in the M-AS patients in our cohort. Specific risk factors for the development of M-AS after taxane administration that may be used a priori for risk stratification of patients are not well characterized. The only established factor, derived from the higher incidence after 3-weekly paclitaxel vs weekly administration, is individual administration dose. ${ }^{7}$ In contrast, cumulative dose does not appear to play a role in this toxicity. Our results are not informative in these particular points, given that we studied patients who received few cycles of docetaxel as part of a single type of regimen in the early breast cancer treatment setting. This was a deliberate decision when designing the protocol to avoid as many confounding factors as possible. Nevertheless, BSA was statistically significantly higher in the M-AS group, and this has implications for the actual dose administered. Several other disease and patient baseline characteristics were examined for possible associations with the M-AS as detailed in the results, but none was confirmed. Concomitant diseases that may predispose to peripheral neuropathy or musculoskeletal pain such as diabetes mellitus and rheumatologic diseases were also not associated with the appearance of the syndrome, and the same was the case for the use of statins, drugs known to predispose to myopathy.
It should be mentioned that the analysis on rheumatologic diseases was somewhat limited, given that patients with such conditions had to have their pain controlled before the period of docetaxel treatment, as, based on the study exclusion criteria, patients with significant ongoing pain have been excluded. Another limitation of our study is that the ESAS pain questionnaire was completed just before administration of the next cycle of chemotherapy, and therefore, pain could have been underreported, given that patients were more than 2 weeks from the time of the usual peak pain occurrence, with a resulting recall bias.

Various treatments have been proposed for taxaneassociated M-AS including corticosteroids, NSAIDs, amifostine, melatonin, glutathione, and the Japanese herbal drug shakuyaku-kanzo-to. ${ }^{8}$ All these held some initial promise, but the overall literature evidence for their efficacy is controversial or negative. A more promising treatment consists of the two newer atypical antiepileptics, gabapentin and pregabalin. Both are used for neuropathic pain including chemotherapyassociated peripheral neuropathy, and anecdotal evidence suggests that they are effective for M-AS. Surprisingly, no published evidence exists for pregabalin, whereas the evidence for gabapentin consists of a case report of two patients and a small series of ten patients. ${ }^{2,3}$ Eleven of these 12 patients had an improvement or complete disappearance of the syndrome with gabapentin treatment. In contrast, the single patient treated with gabapentin in our series did not have a positive effect, in contrast to two patients treated with pregabalin. Prophylactic use of these two medications may have been effective in three additional patients using them for other reasons. Thus, pregabalin or gabapentin may be alternatives to opioid or protracted corticosteroid use, which seem also to be effective but have adverse effects such as constipation and immunosuppression, which can be particularly problematic in chemotherapy patients.

\section{Conclusion}

Overall, our data suggest that a higher BSA may be a risk factor for the development of M-AS. No other clinical parameters examined could elucidate which individual patients would develop the syndrome. It is possible that specific metabolites of taxanes, related to involved enzyme polymorphisms and affecting the way individual patients handle the drugs, could be involved in producing the toxicity. This is a subject for future studies, with a higher number of included patients that will allow for evaluation of polymorphisms effect on M-AS incidence. Given that our results are retrospective, with all the limitations and possible bias that this 
type of data may have, future prospective studies are needed for confirming effectiveness of treatments for the M-AS, not only to improve the quality of life of taxane-treated patients but also to improve oncologic outcomes by keeping these patients on treatment.

\section{Acknowledgments}

The study was supported by a grant from the Sault Ste. Marie Academic Medical Association, Ontario, Canada (to IAV). Data were presented in part in a poster form at European Breast Cancer Conference, Amsterdam, the Netherlands, March 2016.

\section{Disclosure}

The authors report no conflicts of interest in this work.

\section{References}

1. Rowinski EK. Antimicrotubule agents. In: Chabner BA, Longo DL, editors. Cancer Chemotherapy and Biotherapy. Philadelphia, PA: Lippincott Williams and Wilkins; 2006:240-253.

2. Nguyen VH, Lawrence HJ. Use of gabapentin in the prevention of taxaneinduced arthralgias and myalgias. J Clin Oncol. 2004;22:1767-1769.

3. van Deventer H, Bernard S. Use of gabapentin to treat taxane-induced myalgias. J Clin Oncol. 1999;17:434-435.

4. Chang VT, Hwang SS, Feuerman M. Validation of the ESAS. Cancer. 2000;88:2164-2171.

5. Han YM, Smith MT. Pathobiology of cancer chemotherapy-induced peripheral neuropathy (CIPN). Front Pharmacol. 2013;4:156.

6. Reeves BN, Dakhil SR, Sloan JA, et al. Further data supporting that paclitaxel-associated acute pain syndrome is associated with development of peripheral neuropathy. Cancer. 2012;118:5171-5178.

7. Garrison, JA, Mccune, JS, Livingston, et al. Myalgias and arthralgias associated with paclitaxel. Oncology (Williston Park). 2003;17:271-277.

8. Imai A, Matsunami K, Takagi H, Ichigo S. Proposed medications for taxane-induced myalgia and arthralgia (Review). Oncol Lett. 2012;3: $1181-1185$.
Breast Cancer - Targets and Therapy

\section{Publish your work in this journal}

Breast Cancer - Targets and Therapy is an international, peerreviewed open access journal focusing on breast cancer research, identification of therapeutic targets and the optimal use of preventative and integrated treatment interventions to achieve improved outcomes, enhanced survival and quality of life for the cancer patient.

\section{Dovepress}

The manuscript management system is completely online and includes a very quick and fair peer-review system, which is all easy to use. Visit http://www.dovepress.com/testimonials.php to read real quotes from published authors. 